\title{
Effect of Constituent Units, Type of Interflavan Bond, and Conformation on the Antioxidant Properties of Procyanidin Dimers: A Computational Outlook
}

\author{
Ana María Mendoza-Wilson and René Renato Balandrán-Quintana \\ Coordinación de Tecnología de Alimentos de Origen Vegetal, CIAD, A.C., Carretera a la Victoria Km 0.6, \\ 83304 Hermosillo, SON, Mexico \\ Correspondence should be addressed to Ana María Mendoza-Wilson; mwilson@ciad.mx
}

Received 1 December 2016; Accepted 23 January 2017; Published 21 February 2017

Academic Editor: Artur M. S. Silva

Copyright (C) 2017 Ana María Mendoza-Wilson and René Renato Balandrán-Quintana. This is an open access article distributed under the Creative Commons Attribution License, which permits unrestricted use, distribution, and reproduction in any medium, provided the original work is properly cited.

\begin{abstract}
Procyanidin (PC) dimers are powerful antioxidants, abundant in plant tissues, and also bioavailable. However, the role of the molecular structure of PCs on their antioxidant properties is still a controversial and not fully understood issue that needs to be addressed in a more specific way. The objective of this study was to analyze the effect of the constituent units, type of interflavan bond, and conformation on the antioxidant properties of PC dimers including PB3, PB4, PB5, PB6, PB7, and PB8, using the density functional theory (DFT) computational method. The analysis was performed in function of parameters that allow determining the ability of the molecules to transfer or to capture electrons, among which the chemical potential, bond dissociation enthalpy (BDE), gap energy, Fukui indices, and charge distribution of HOMO-LUMO orbitals. The factors that showed the most notable effects on the antioxidant properties of the PC dimers were the type of interflavan bond and the conformation. The antioxidant ability of the dimers PB3 and PB4 containing the interflavan bond C4-C8, in their Compact conformation, was very similar to each other but greater than those of dimers PB5, PB6, PB7, and PB8 containing the C4-C6 interflavan bond. PB8 showed the lowest antioxidant ability.
\end{abstract}

\section{Introduction}

Procyanidins (PCs) are recognized as potent antioxidants due to their ability to inhibit the detrimental effects of oxidative enzymes, metals, and free radicals. These compounds are considered bioresources with possible applications in food, medicine, and pharmacology [1]. However, the relationship between the molecular structure of PCs and their antioxidant properties is still a controversial and not fully understood issue that needs to be addressed in a more specific way. This is in part due to the great structural diversity of the PCs regarding the degree of polymerization, type of constitutive units, type of interflavan bond, and conformations.

The PCs are oligomeric or polymeric derivatives of flavan3 -ols, which are constituted by (+)-catechin and/or (-)epicatechin units, mainly linked through C4-C8 or C4-C6 interflavan bonds. When the interflavan bond is positioned ahead of ring $\mathrm{C}$, it is called $\beta$; but when this is oriented backward of ring $\mathrm{C}$, it is appointed as $\alpha$. The upper units of the chain are denominated extension units (EU) and the end unit is known as terminal unit (TU) [2]. Since there are several chiral centers (C2, C3, and C4) in these molecules, they can exist as a variety of stereoisomers. Due to the flexibility of ring $\mathrm{C}$, the compact (Com) and extended (Ext) conformations are formed (Figure 1) [3]. In the conformation Com, the two rings $B$ are opposite to each other, whereas they are facing each other in the conformation Ext [4].

The PCs are abundant in fruits, cereals, legumes, and their derived processed foods. Approximately $40 \%$ of the PCs ingested in the diet correspond to monomers, dimers, trimers, and tetramers $[5,6]$. The dimers are not only abundant but also bioavailable, so that some common dimers in plan tissues as PB1 [(-)-epicatechin- $(\mathrm{C} 4 \beta \rightarrow \mathrm{C} 8)-(+)$-catechin] and PB2 [(-)-epicatechin- $(\mathrm{C} 4 \beta \rightarrow \mathrm{C} 8)-(-)$-epicatechin] have 

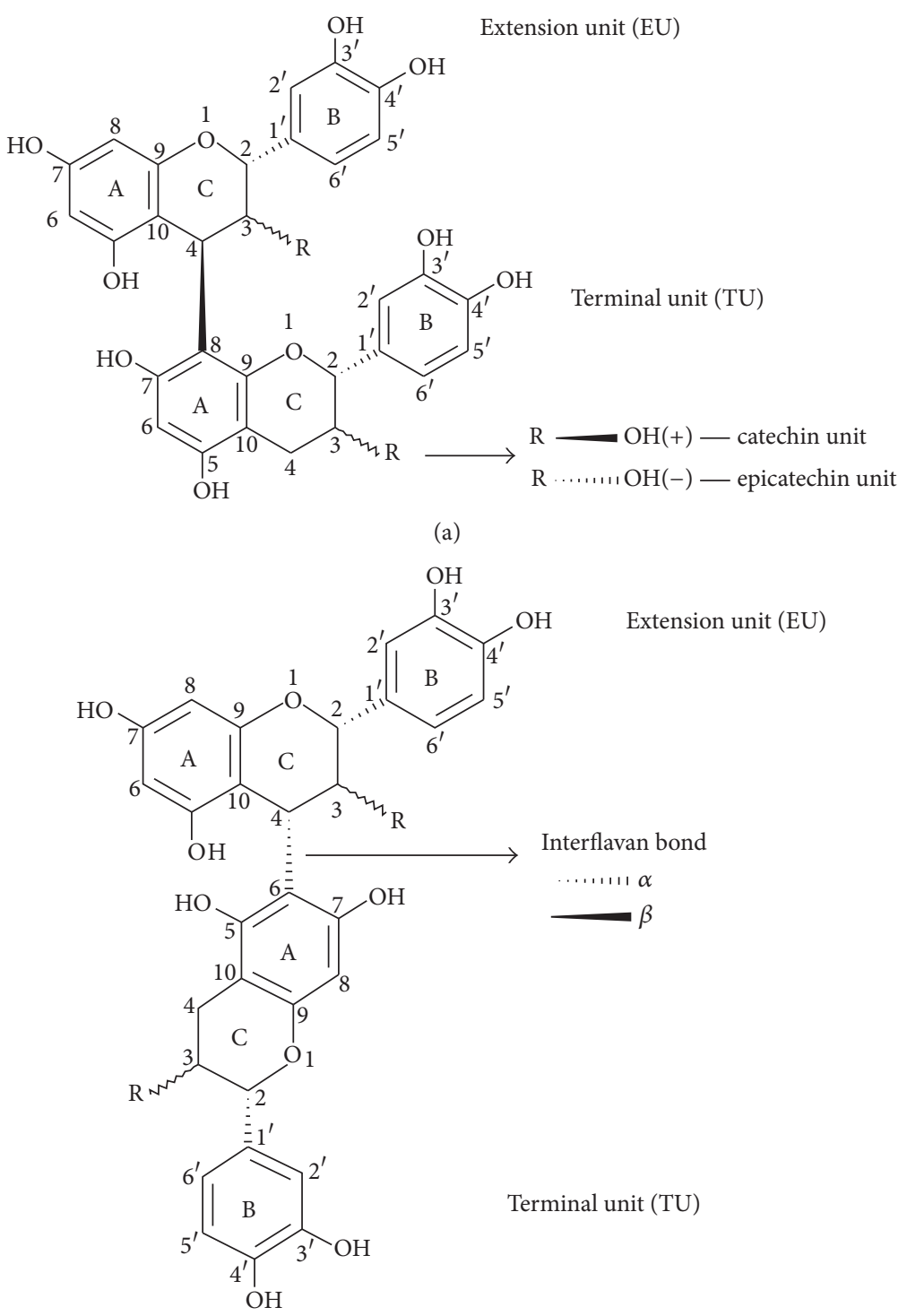

(b)

FIGURE 1: General structure of procyanidin dimers with interflavan bond types C4-C8 and C4-C6. (a) PB3 (C4 $\alpha-\mathrm{C} 8$, catechin); PB4 (C4 $\alpha-$ C8, catechin-epicatechin). (b) PB5 (C4 $\beta-\mathrm{C} 6$, epicatechin); PB6 (C4 $\alpha-\mathrm{C} 6$, catechin); PB7 (C4 $\beta-\mathrm{C} 6$, epicatechin-catechin); PB8 (C4 $\alpha-\mathrm{C} 6$, catechin-epicatechin).

been preferentially studied. Other PC dimers, including PB3 $[(+)$-catechin-(C4 $\alpha \rightarrow \mathrm{C} 8)-(+)$-catechin $], \mathrm{PB} 4[(+)$-catechin$(\mathrm{C} 4 \alpha \rightarrow \mathrm{C} 8)-(-)$-epicatechin], PB5 [(-)-epicatechin- $(\mathrm{C} 4 \beta \rightarrow$ C6)-(-)-epicatechin $], \quad$ PB6 $[(+)$-catechin- $(\mathrm{C} 4 \alpha \rightarrow \mathrm{C} 6)-(+)-$ catechin] PB7 $[(-)$-epicatechin- $(\mathrm{C} 4 \beta \rightarrow \mathrm{C} 6)-(+)$-catechin], and PB8 $[(+)$-catechin- $(\mathrm{C} 4 \alpha \rightarrow \mathrm{C} 6)-(-)$-epicatechin $]$, are less frequent in foods but are important components of fruits and cereals of high commercial value, among these grape, apple, and barley, as well as derived beverages, such as juice, red wine, and beer [7-9]. Together, these dimers afford contrasting structural characteristics related to the type of constituent units, type of interflavan bond, and conformation, whose study could provide insights concerning the role of molecular structure of PCs on their antioxidant properties. Previously, De Freitas et al. [7] performed an experimental-computational study that addressed the relationship between molecular structure and susceptibility to oxidation of those dimeric PCs. However, data relating the electron transfer and electron density changes were not taken into account.

The antioxidant reactions operate through electron transfer from a donor molecule (PCs) to an acceptor one (enzyme, metal, and free radical). The electron transfer process can occur by different ways: either directly or in the form of hydrogen atoms; by combination with molecular oxygen or, even, by using electron transporters $\left(\mathrm{NAD}^{+}, \mathrm{NADP}^{+}, \mathrm{FAD}\right.$, FMN) [10]. The antioxidant reactions also can occur through nucleophilic or electrophilic attack.

Since molecules reactivity depends on its structure, the first step to explore the antioxidant properties of compounds 
of interest is to know the most susceptible molecular sites for donating and accepting electrons, as well as the relative stabilities of the molecules.

Computational chemistry is a useful tool for studying the reactivity properties of compounds, especially those with a great structural diversity like the PCs. Through a computational chemistry approach, diverse analysis can be executed which offer a better sight of the effect of structure on the antioxidant properties. In relation to the type of constituent units, type of interflavan bond, and conformation, parameters such as the dihedral angles of the interflavan bond, intramolecular hydrogen bonds between monomeric units, molecular surface area, and stability of the PCs may be of great relevance. Regarding the capacity for transferring electrons, the chemical potential is essential, as well as thermochemical parameters such as bond dissociation enthalpy (BDE), gap energy, Fukui indices, and charge distribution of HOMO-LUMO orbitals to determine sites of nucleophilic and electrophilic attack.

The density functional theory (DFT) is among the computational methods which offer a high accuracy for simulating distinct conformations and exploring properties based on electron transfer, besides changes in electron density in complex molecular systems.

The objective of this study was to analyze the effect of the constituent units, type of interflavan bond, and conformation on the antioxidant properties of PCs dimers including PB3, PB4, PB5, PB6, PB7, and PB8, employing the DFT method.

\section{Computational Methods}

The ground-state molecular geometries, harmonic vibrational frequencies, and reactivity calculations were performed under the simulation of an aqueous medium, within the framework of DFT method implemented in the GAUSSIAN 09W computational package [11].

The M05-2X/6-31G ${ }^{* *}$ model chemistry in combination with the SMD solvation method was selected for this study because they are considered appropriate for the type of variables to be analyzed. The M05-2X is a highly parameterized hybrid metaexchange-correlation functional, which yields satisfactory results for thermochemistry parameters and noncovalent interactions (hydrogen bonding) [12, 13]. On the other hand, the SMD model is applicable to any charged or uncharged solute molecule interacting with a continuum solvent. The elements present in the solutes included $\mathrm{H}, \mathrm{C}$, and $\mathrm{O}$, which are part of the PCs. The SMD model employs a single set of parameters, among them the intrinsic atomic Coulomb radii and atomic surface tension coefficients, optimized over six electronic structure methods, within which are included M05-2X/6-31G ${ }^{*}$ and M05-2X/6$31+\mathrm{G}^{* *}$. With the use of $6-31 \mathrm{G}^{*}$ basis set, the SMD model achieves mean errors of $0.6-1.0 \mathrm{kcal} / \mathrm{mol}$ in the solvation free energies of neutral molecules and mean errors around of $4 \mathrm{kcal} / \mathrm{mol}$ for ions [14].

For a better understanding of the effect of the type of constituent units, type of interflavan bond, and conformation on the antioxidant properties of PC dimers, parameters such as the dihedral angles of interflavan bond, intramolecular hydrogen bonds between monomeric units, the molecular surface area, and stability of the PCs were determined.

The dihedral angles of $\mathrm{PC}$ dimers were measured among $\mathrm{C} 3-\mathrm{C} 4-\mathrm{C} 8-\mathrm{C} 9$ and $\mathrm{C} 3-\mathrm{C} 4-\mathrm{C} 6-\mathrm{C} 7$ carbon atoms for $\mathrm{C} 4-\mathrm{C} 8$ and C4-C6 interflavan bonds, respectively.

To know the molecular surface area of the PC dimers, a density cube file was generated from the optimized geometries in Gaussian 09; then the Chimera software 1.11rc [15] was used to read the cube data file and the surface area of PC dimers was determined at \pm 0.001 a.u. isodensity value.

To estimate the stability of the PC dimers, the Gibbs free energy including the thermal free energy corrections $\left(E_{0}+\right.$ $G_{\text {corr }}$ ) was obtained as one of the thermochemical values, computed in the Gaussian 09 program from a vibrational frequency calculation in aqueous medium, using the rigidrotor harmonic-oscillator approximation (RRHO) at $298 \mathrm{~K}$ [16]. The stability of the PC dimers was established as a relative value of free energy $(\Delta E)$, that is, as the difference in free energy between the lowest-energy dimer and the remaining dimers in their distinct conformations.

The calculated reactivity properties included those parameters that define the chemical potential, such as ionization potential, electron affinity, hardness, electronegativity, and electrophilicity; also, the HOMO-LUMO gap energies and thermochemical parameters such as bond dissociation enthalpy (BDE), Fukui indices, and charge distribution of HOMO-LUMO orbitals were included.

The parameters of the chemical potential were obtained from the differences of total electronic energies when adding or removing an electron, in relation to the neutral molecule, using a vertical approach that is without reoptimization of the molecular geometries [17]. The equations used to calculate these parameters are as follows:

Ionization potential is

$$
I=\text { cation energy }- \text { neutral molecule energy. }
$$

Electron affinity is

$$
A=\text { neutral molecular energy }- \text { anion energy. }
$$

Hardness is

$$
\eta=\frac{1}{2}(I-A)
$$

Electronegativity is

$$
\chi=\frac{1}{2}(I+A) .
$$

Electrophilicity is

$$
\omega=\frac{\mu^{2}}{2 \eta},
$$

where $\mu=-\chi$, the negative of electronegativity $(\chi)$ is equivalent to chemical potential $(\mu)$.

The HOMO-LUMO gap energies were calculated as the difference in energy between the frontier orbitals HOMO and LUMO, as indicated in the following equation [18]:

$$
\text { gap }=E_{\mathrm{LUMO}}-E_{\mathrm{HOMO}} .
$$


In order to know the ability of the PC dimers to transfer electrons in form of hydrogen atoms, the bond dissociation enthalpy (BDE) was calculated. This was determined as the difference of enthalpy between parent molecule $(\mathrm{ArOH})$ and its phenoxyl radical $\left(\mathrm{ArO}^{\bullet}\right)$ formed after the hydrogen atom $\left(\mathrm{H}^{\circ}\right)$ transfer from each phenolic or hydroxyl group $(\mathrm{OH})$. The reaction (7) and (8) were applied [19]:

$$
\begin{aligned}
\mathrm{ArOH} \longrightarrow & \mathrm{ArO}+\mathrm{H}^{\bullet} \\
\Delta_{r} H(298 \mathrm{~K})= & \Sigma\left(E_{0}+H_{\text {corr }}\right)_{\text {products }} \\
& -\Sigma\left(E_{0}+H_{\text {corr }}\right)_{\text {reactants }} .
\end{aligned}
$$

The computed enthalpy of hydrogen atom $\left(\mathrm{H}^{\circ}\right)$ in aqueous phase was -0.493267 Hartrees.

The Fukui function is a local property derived from the electronic density and is defined by the following relation:

$$
f(r)=\left(\frac{\partial \rho(r)}{\partial N}\right)_{v(r)},
$$

where $\rho$ represents the electronic density of the system under consideration [20].

The condensed Fukui functions are found by taking the finite difference approximations from charge population analysis of atoms in molecules, depending on the direction of the electron transfer; for nucleophilic and electrophilic attack, the functions are described as follows [21]:

$$
\begin{aligned}
& f_{k}^{+}=q_{k}(N+1)-q_{k}(N) \text { (nucleophilic attack) } \\
& f_{k}^{-}=q_{k}(N)-q_{k}(N-1)(\text { electrophilic attack), }
\end{aligned}
$$

where $q_{k}$ is the gross charge of atom $k$ in the molecule.

The charge distributions of HOMO and LUMO orbitals were visualized from the optimized geometries of PC dimers in aqueous medium by Gauss View 5.0.9 program.

\section{Results and Discussion}

3.1. Molecular Properties and Stability of the PC Dimers. Previous studies have shown that PC dimers can acquire two energy-minimized structures, which are identified based on the dihedral angle. One of the optimized structures is characterized by adopting a conformation Com, while the others adopt a conformation Ext [4, 22].

The dihedral angles established among the C3-C4-C8C9 or C3-C4-C6-C7 carbon atoms of the PC dimers optimized in aqueous medium are displayed in Table 1. PB3 and $\mathrm{PB} 4$ have a $\mathrm{C} 4 \alpha-\mathrm{C} 8$ interflavan bond, so they can acquire Com and Ext conformations; therefore the dihedral angles formed in both conformations are shown. Nonetheless, in $\mathrm{PB} 5, \mathrm{~PB} 6, \mathrm{~PB} 7$, and $\mathrm{PB} 8$, which possess $\mathrm{C} 4 \alpha-\mathrm{C} 6$ or $\mathrm{C} 4 \beta-\mathrm{C} 6$ interflavan bonds, only one conformation can be adopted in its ground-state. In the case of PB5 and PB7, which have $\mathrm{C} 4 \beta-$ C6 interflavan bond (ahead of ring C), and (-)-epicatechin as extension unit, a conformation Com was acquired, while, in PB6 and PB8 with C4 $\alpha-\mathrm{C} 6$ interflavan bond (backward of ring $\mathrm{C}$ ) and (+)-catechin as extension unit, a conformation Ext was adopted.
It is important to clarify that the dihedral angles obtained from the energy-minimized structures of the PC dimers do not come from calculations of torsional profiles from $0^{\circ}$ to $360^{\circ}$. The way we check that the structures correspond to absolute minima or true minima was calculating the force constants and vibrational frequencies on the stationary points obtained after the optimization of PC dimers. With such data, the presence of imaginary frequencies (negative values) was discarded and it was verified that the forces representing the minima remained constant in all directions of the molecular plane. For the last point, four criteria that confirm convergence were taken into account [11] and these are as follows:

(1) The maximum component of the force must be below the cutoff value of 0.00045 in all directions of the molecular plane.

(2) The root-mean-square (RMS) of the forces must be below the defined tolerance of 0.0003 .

(3) The calculated displacement for the next step must be smaller than the defined cutoff value of 0.0018 .

(4) The RMS of the displacement for the next step must be below its cutoff value of 0.0012 .

The three-dimensional orientation of PC dimers induced by the adopted conformations (Com or Ext) can lead to the formation of intramolecular hydrogen bonds (IHBs) between their constituent units. These bonds exert important effects on the interactions that the conformers may establish with other molecules such as oxidants or solvents. The analysis of interatomic bond distances of the PC dimers optimized in aqueous medium suggest that, in PB3 Com, PB4 Com, PB5, PB6, PB7, and PB8, IHBs were not formed. Instead, the conformation Ext of PB3 and PB4 can favor the formation of IHBs between the catechol moieties (B rings) of the extension and terminal units, with values within a range from 2.0 to $2.53 \AA$ (Figure 2).

With the purpose of corroborating the establishment of these IHBs, the O-H stretching frequencies of the catechol moieties were analyzed, and these appear around $3700 \mathrm{~cm}^{-1}$ in PB3 Ext and PB4 Ext. The literature indicates that, in orthosubstituted phenols, which can form IHBs, it is possible to locate the $\mathrm{O}-\mathrm{H}$ stretch band in the region of 3670$3607 \mathrm{~cm}^{-1}$ in vapor phase and this band could be shifted to higher frequencies in dilute solutions. The abnormally high $\mathrm{O}-\mathrm{H}$ stretching frequencies are attributed to the repulsion between $\mathrm{O}-\mathrm{H}$ groups and protons of other groups [23]. In the case of PB3 Com, PB4 Com, PB5, PB6, PB7, and PB8, the $\mathrm{O}-\mathrm{H}$ stretching frequencies including the catechol moieties were detected in the range of $3900-3800 \mathrm{~cm}^{-1}$ region, where the free $\mathrm{O}-\mathrm{H}$ stretching in phenols can be observed.

The above results are different to data reported in other studies, in which IHBs were favored in conformation Com but not in the conformation Ext of distinct PC oligomers linked by $\mathrm{C} 4 \beta-\mathrm{C} 8$ interflavan bonds, among which are the $\mathrm{PB} 1$ and $\mathrm{PB} 2$ dimers, $\mathrm{PC} 1$ trimer, and a B-type tetramer [24, 25]. These results highlight the importance of the extension unit monomer and the atoms involved in the interflavan 
TABLE 1: Molecular properties and stability $(\Delta E)$ of the procyanidin dimers optimized in aqueous medium (SMD model) using the M05$2 \mathrm{X} / 6-31 \mathrm{G}^{* *}$ DFT method.

\begin{tabular}{lccc}
\hline Procyanidin dimer & Dihedral angle (degrees) & $\begin{array}{c}\text { Molecular surface area } \\
\left(\AA^{2}\right)\end{array}$ & $\begin{array}{c}\text { Relative energy } \\
(\Delta E \mathrm{kcal} / \mathrm{mol})\end{array}$ \\
\hline PB3 Com & $114.20(\mathrm{C} 3-\mathrm{C} 4-\mathrm{C} 8-\mathrm{C} 9)$ & 745.9 & 3.49 \\
PB3 Ext & $-35.39(\mathrm{C} 3-\mathrm{C} 4-\mathrm{C} 8-\mathrm{C} 9)$ & 678.5 & 6.49 \\
PB4 Com & $102.08(\mathrm{C} 3-\mathrm{C} 4-\mathrm{C} 8-\mathrm{C} 9)$ & 744.4 & 0 \\
PB4 Ext & $-65.12(\mathrm{C} 3-\mathrm{C} 4-\mathrm{C} 8-\mathrm{C} 9)$ & 677.3 & 0.23 \\
PB5 & $101.72(\mathrm{C} 3-\mathrm{C} 4-\mathrm{C} 6-\mathrm{C} 7)$ & 748.9 & 5.05 \\
PB6 & $-59.32(\mathrm{C} 3-\mathrm{C} 4-\mathrm{C} 6-\mathrm{C} 7)$ & 751.4 & 3.12 \\
PB7 & $101.37(\mathrm{C} 3-\mathrm{C} 4-\mathrm{C} 6-\mathrm{C} 7)$ & 745.3 & 4.63 \\
PB8 & $-59.49(\mathrm{C} 3-\mathrm{C} 4-\mathrm{C} 6-\mathrm{C} 7)$ & 749.8 & 4.55 \\
\hline
\end{tabular}

PB3 (C4 $\alpha-C 8$, catechin); PB4 (C4 $\alpha-C 8$, catechin-epicatechin); PB5 (C4 $\beta-C 6$, epicatechin); PB6 (C4 $\alpha-C 6$, catechin); PB7 (C4 $\beta-C 6$, epicatechin-catechin); PB8 (C4 $\alpha-\mathrm{C} 6$, catechin-epicatechin).

$\Delta E$ was calculated as the difference in free energy between the lowest-energy dimer and the remaining dimers.

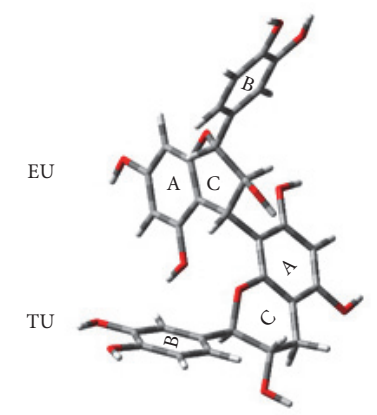

PB3 Com

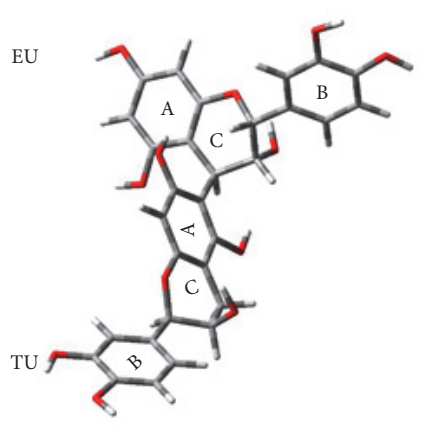

PB5

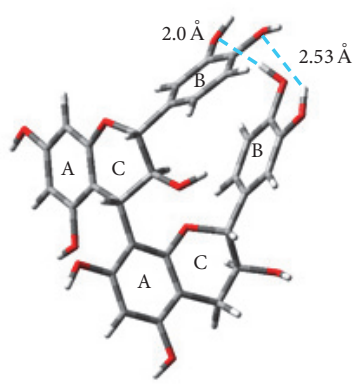

PB3 Ext

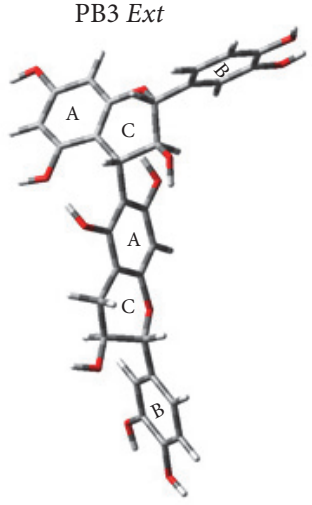

PB6
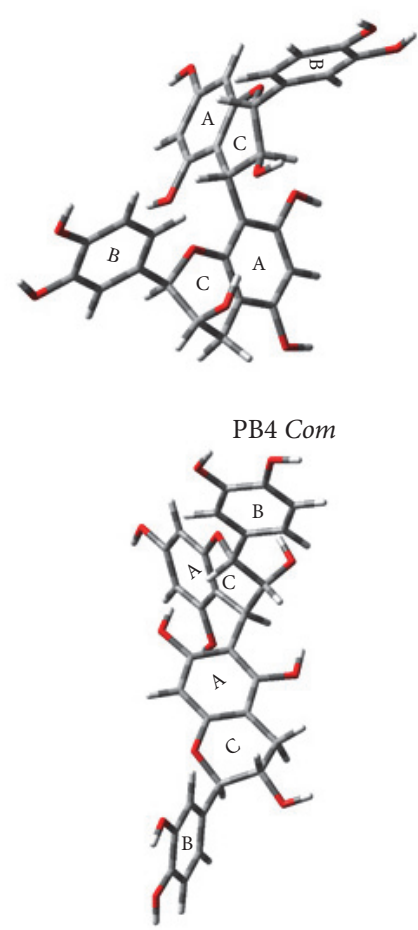

PB7
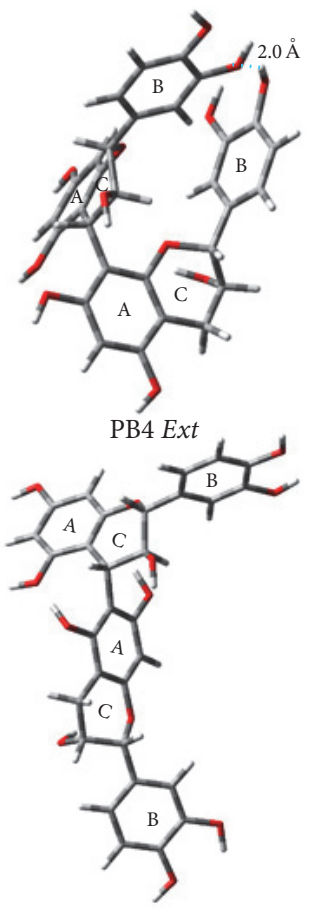

PB8

FIGURE 2: Optimized geometry of the procyanidin dimers in aqueous medium (SMD model) using the M05-2X/6-31G ${ }^{* *}$ DFT method. Com: compact conformation; Ext: extended conformation.

bond (C4-C8 or $\mathrm{C} 4-\mathrm{C} 6)$, as well as the bond orientation ( $\alpha$ or $\beta$ ). Those that can favor the formation of IHBs are the joins $\mathrm{C} 4-\mathrm{C} 8$ but not the $\mathrm{C} 4-\mathrm{C} 6$. When the extension unit is (-)-epicatechin and the interflavan bond acquires the $\beta$ orientation $(\mathrm{C} 4 \beta-\mathrm{C} 8)$, IHBs in conformation Com can be promoted, but if the extension unit is $(+)$-catechin and the interflavan bond acquires the $\alpha$ orientation $(\mathrm{C} 4 \alpha-\mathrm{C} 8)$, IHBs in conformation Ext could be favored.

The conformation Ext is characterized by a rearrangement where the two rings $B$ are facing each other. Depending on the proximity of these catechol rings, an intramolecular $\pi-\pi$ stacking arrangement can be adopted leading to the formation of IHBs, which reduces the total surface of the molecule [4]. Considering the repercussion that this could have on the accessibility of the solvent or oxidizing agents in the reactive sites of the molecules, the molecular surface area of PC dimers was measured.

In Table 1, it can be seen that PB5, PB6, PB7, and PB8 with $\mathrm{C} 4-\mathrm{C} 6$ interflavan bond showed the greatest molecular surface areas (745 to $751 \AA^{2}$ ), whereas PB3 and PB4 with C4C8 interflavan bond in their conformation Ext showed the smallest molecular surface areas $\left(677\right.$ to $\left.678 \AA^{2}\right)$. Figure 3 depicts the total electronic density of the molecules of PB6, PB3 Ext, and PB4 Ext, in order to visualize the difference 


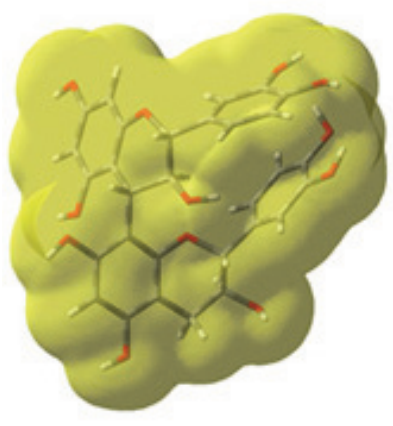

PB3 Ext

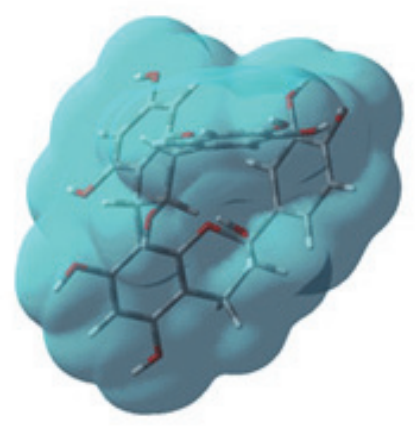

PB4 Ext

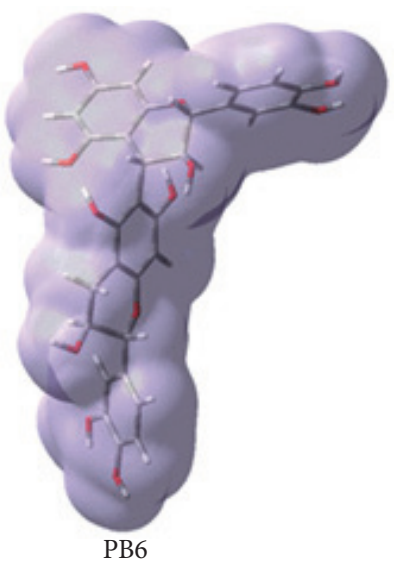

Figure 3: Molecular surface area of the PB3 Ext, PB4 Ext, and PB6 procyanidin dimers, determined under simulation of an aqueous medium (SMD model) using the M05-2X/6-31G** DFT method. Ext: extended conformation.

between the molecule with greater surface area (PB6) and the molecules with smaller surface area (PB3 Ext and PB4 Ext).

Regarding the stability of PC dimers, PB4 was the one that exhibited the most negative values of free energy in aqueous medium, mainly in the conformation Com followed by the conformation Ext, indicating that it was the most stable dimer. The relative free energy $(\Delta E)$ between conformations Com and Ext of PB4 was $0.23 \mathrm{kcal} / \mathrm{mol}$, suggesting that they could be exchanged easily (Table 1). However, in a study involving nuclear magnetic resonance and molecular modeling, it was reported that, in PB4, the conformation Com predominates in aqueous ethanol solution [7]. In another similar study, it was found that, in PB2 dimer, which has (-)epicatechin as terminal unit such as PB4, even proportions of Com and Ext conformations were detected when it was dissolved in water and hydroalcoholic media [26].

The relative stability of the dimers based on the lowest values of $\Delta E$ and taking PB4 as reference because of being the most stable was as follows (Table 1 ).

PB4 Com $(0 \mathrm{kcal} / \mathrm{mol})>$ PB4 Ext $(0.23 \mathrm{kcal} / \mathrm{mol})>\mathrm{PB} 6$ (3.12 kcal $/ \mathrm{mol})>$ PB3 Com $(3.49 \mathrm{kcal} / \mathrm{mol})>$ PB8 $(4.55 \mathrm{kcal} /$ $\mathrm{mol})>$ PB7 $(4.63 \mathrm{kcal} / \mathrm{mol})>$ PB5 (5.05) > PB3 Ext $(6.49 \mathrm{kcal} /$ $\mathrm{mol})$.

As can be appreciated in Table 1, PB3 in its conformation Ext is the less stable dimer even establishing two IHBs between the B rings of both extension and terminal units. On the one hand, this can be attributed to the fact that the dihedral angle of the interflavan bond is small $(-35.39)$ and generates steric hindrance among the remaining $\mathrm{O}-\mathrm{H}$ groups by the proximity of the aromatic rings. On the other hand, it is important to take into account the fact that all molecules have an effective surface area, which is determined by the conformation, and this is more important for the interactions with solvent than the total surface area. In addition to the formation of IHBs, the establishment of intermolecular hydrogen bonds with the solvent can contribute to the stability of molecules in solution and marking the differences of solvation free energy among PC dimers. To this respect, it is important to mention that, in the SMD solvation model, the interactions of the solute and the surrounding solvent do not take into account the molecular structure of the solvent and that the dielectric response of the medium is uniform and linear at all positions outside the space that defines the solute. For this reason, the SMD model does not provide a completely valid description neither of the solvent structure nor of the solute-solvent interactions (hydrogen bonding, $\pi$ $\pi$ stacking interactions). However, the SMD model allows estimating the geometry-dependent proportionality constants called atomic surface tensions that describe the solventaccessible surface areas of the individual atoms of the solute [14]. On these bases, it is considered that the SMD solvation model could give an approximate and acceptable description of the relative stabilities of the PC dimers in their distinct conformations.

3.2. Chemical Potential, Gap Energy, and Stability of Anions and Cations. The chemical potential is defined by a set of parameters which measure the tendency to transfer or capture electrons and therefore describe the antioxidant properties of the molecules. The parameters that determine the ability to transfer electrons are the ionization potential $(I)$ and hardness $(\eta)$. I represents the amount of energy required to remove an electron of a molecule and $\eta$ is a measure of the resistance to charge transference [18]. On the other hand, the ability to capture electrons is determined by the electron affinity $(A)$, electronegativity $(\chi)$, and electrophilicity $(\omega)$. A is defined as the energy release when an electron is added to a neutral molecule, while $\chi$ indicates the tendency to attract electrons and $\omega$ establishes the decay of binding energy due to a maximum electron flow between a donor and a receptor [18].

PCs, like all flavonoids, have atomic regions that allow them to easily transfer electrons but also have other atomic regions through which they react accepting electrons from other compounds. Molecules with improved antioxidant properties are those that readily transfer the electrons and have a low capacity to capture them, that is, having low values of $I, A, \eta, \chi$, and $\omega$. It is known that a large value of $A$ and $\omega$ 
TABLE 2: Parameters of chemical potential and gap energy of the procyanidin dimers calculated by the M05-2X/6-31G ${ }^{* *}$ DFT method in aqueous medium (SMD model).

\begin{tabular}{|c|c|c|c|c|c|c|}
\hline Procyanidin dimer & $I(\mathrm{eV})$ & $A(\mathrm{eV})$ & $\eta(\mathrm{eV})$ & $\chi(\mathrm{eV})$ & $\omega(\mathrm{eV})$ & Gap $(\mathrm{eV})$ \\
\hline PB3 Com & 5.84 & 0.28 & 2.78 & 3.06 & 1.68 & 6.10 \\
\hline PB3 Ext & 6.01 & 0.39 & 2.81 & 3.20 & 1.83 & 6.31 \\
\hline PB4 Com & 5.82 & 0.31 & 2.76 & 3.06 & 1.70 & 6.11 \\
\hline PB4 Ext & 5.84 & 0.40 & 2.72 & 3.12 & 1.79 & 6.27 \\
\hline PB5 & 5.95 & 0.34 & 2.81 & 3.15 & 1.77 & 6.37 \\
\hline PB6 & 5.99 & 0.29 & 2.85 & 3.15 & 1.74 & 6.27 \\
\hline PB7 & 5.92 & 0.28 & 2.82 & 3.09 & 1.70 & 6.34 \\
\hline PB8 & 6.34 & 0.32 & 3.01 & 3.33 & 1.84 & 6.32 \\
\hline
\end{tabular}

PB3 (C4 $\alpha-C 8$, catechin); PB4 (C4 $\alpha-C 8$, catechin-epicatechin); PB5 (C4 $\beta-C 6$, epicatechin); PB6 (C4 $\alpha-C 6$, catechin); PB7 (C4 $\beta-C 6$, epicatechin-catechin); PB8 (C4 $\alpha-$ C6, catechin-epicatechin).

$I$ : ionization potential; $A$ : electron affinity; $\eta$ : hardness; $\chi$ : electronegativity; $\omega$ : electrophilicity.

may lead to prooxidant effects and a decrease in antioxidant ability [27, 28].

As displayed in Table 2, the chemical potential parameters fluctuated within a comparable range of values in all PC dimers: $I(5.82-6.34 \mathrm{eV}), A(0.28-0.40 \mathrm{eV}), \eta(2.72-$ $3.01 \mathrm{eV}), \chi(3.06-3.33 \mathrm{eV}), \omega(1.68-1.84 \mathrm{eV})$. These values are lower than those reported for quercetin [29], I (7.22 eV), A $(0.76 \mathrm{eV}), \eta(3.23 \mathrm{eV}), \chi(3.99 \mathrm{eV}), \omega(2.47 \mathrm{eV})$, which could mean that the PC dimers possess high antioxidant ability, since quercetin is considered one of the flavonoids with greater antioxidant activity.

Notwithstanding the previously discussed, differences were observed among the analyzed PC dimers. In general terms, the dimers PB3 and PB4 in their conformation Com were the ones that showed the lowest values for all the variables associated with the chemical potential, while the dimer PB8 presented the highest values (Table 2).

The band gap represents the conductive layer of electrons in a molecule and is determined by the energy difference between the valence orbitals HOMO and LUMO. Reactive molecular systems that easily transfer their electrons are associated with low gap values, whereas, in the opposite case, the less reactive molecules have higher gap values $[17,18]$. The gap values obtained in our study, shown in Table 2, suggest that PB3 and PB4 in their conformation Com could be the dimers that more easily transfer their electrons, since the energy difference between HOMO and LUMO was lower (6.10-6.11 eV) in comparison to the PB5, PB6, PB7, and PB8 dimers $(6.27-6.37 \mathrm{eV})$. These results corroborate those found for the chemical potential.

3.3. Bond Dissociation Enthalpy (BDE). One of the most common ways of studying the ability of flavonoids to inhibit free radicals is through thermochemical parameters, among these the BDE, because the electron transfer in the form of hydrogen atoms is one of the preferential mechanisms followed by flavonoids $[24,30]$. In Table 3 , it can be seen that all the analyzed PC dimers showed the same behavior regarding the easiness of each phenolic group for donating its hydrogen atom; the lower the BDE value, the higher the easiness for transferring the hydrogen atom. On these bases, the following general sequence for the distinct $\mathrm{OH}$ groups of the dimers is proposed, in both the extension and terminal units.

Easiness to transfer hydrogen atoms (BDE values) from the $\mathrm{OH}$ groups in the extension unit of $\mathrm{PC}$ dimers: $4^{\prime}-\mathrm{OH}$ $(81.87-83.03 \mathrm{kcal} / \mathrm{mol})>3^{\prime}-\mathrm{OH}(83.85-86.83 \mathrm{kcal} / \mathrm{mol})>5-$ $\mathrm{OH}(88.27-90.64 \mathrm{kcal} / \mathrm{mol})>7-\mathrm{OH}(88.77-92.79 \mathrm{kcal} / \mathrm{mol})$.

Easiness to transfer hydrogen atoms (BDE values) from the $\mathrm{OH}$ groups in the terminal unit of $\mathrm{PC}$ dimers: $4^{\prime}-\mathrm{OH}$ $(82.28-83.33 \mathrm{kcal} / \mathrm{mol})>3^{\prime}-\mathrm{OH}(84.04-86.53 \mathrm{kcal} / \mathrm{mol})>5-$ $\mathrm{OH}(84.72-87.27 \mathrm{kcal} / \mathrm{mol})>7-\mathrm{OH}(85.78-89.07 \mathrm{kcal} / \mathrm{mol})$.

The BDE values displayed above suggest that the $4^{\prime}-\mathrm{OH}$ and $3^{\prime}-\mathrm{OH}$ groups, which constitute the catechol moiety of ring $\mathrm{B}$, show more facility to transfer their hydrogen atoms in both the extension and terminal units.

The phenolic groups that show the greatest facility for transferring their hydrogen atoms (less BDE) are those which confer better antioxidant properties to the molecules. On these bases, it is suggested that the $4^{\prime}-\mathrm{OH}$ group is the most antioxidant site of the PC dimers.

The BDE values of the phenoxyl radicals derived from the $4^{\prime}-\mathrm{OH}$ group of the different dimers were very close to each other; however, these tend to be slightly lower for PB3 and PB4, especially in the conformation Ext, indicating greater antioxidant ability for such dimers compared to PB5, PB6, PB7, and PB8. These results are consistent with those reported by [7], who performed a kinetic study of oxidative susceptibility in the same dimers. The compounds with higher susceptibility to oxidation are those that may present better antioxidant properties by protecting other molecules from oxidation.

3.4. Fukui Indices and Charge Distribution in HOMO and LUMO Orbitals. The Fukui indices calculation was carried out using the Hirshfeld charge population analysis. Through this scheme it is possible to study well-defined atomic fragments, which when they integrated, they allow establishing the contribution of each fragment in the changes of electronic density, in the deformation of the bonds, electrostatic potential, and the transferability of net atomic charge at molecular level [31].

During the reaction of the PCs against different radical species, oxidative enzymes, and metals, these can develop 
TABLE 3: Bond dissociation enthalpy (BDE) of the procyanidin dimers calculated in aqueous medium (SMD model) using the M05-2X/6$31 G^{* *}$ DFT method.

\begin{tabular}{|c|c|c|c|c|c|c|c|c|}
\hline \multirow{3}{*}{ Procyanidin dimer } & \multicolumn{8}{|c|}{$\mathrm{BDE}(\mathrm{kcal} / \mathrm{mol})$} \\
\hline & \multicolumn{4}{|c|}{ Extension unit (EU) } & \multicolumn{4}{|c|}{ Terminal unit (TU) } \\
\hline & $3^{\prime}-\mathrm{OH}$ & $4^{\prime}-\mathrm{OH}$ & $5-\mathrm{OH}$ & 7-OH & $3^{\prime}-\mathrm{OH}$ & $4^{\prime}-\mathrm{OH}$ & $5-\mathrm{OH}$ & 7-OH \\
\hline PB3 Com & 85.51 & 82.36 & 88.57 & 88.77 & 84.04 & 83.03 & 87.27 & 87.21 \\
\hline PB3 Ext & 84.60 & 81.87 & 88.53 & 89.92 & 86.11 & 82.42 & 86.51 & 85.78 \\
\hline PB4 Com & 86.27 & 83.03 & 88.28 & 88.79 & 85.70 & 82.42 & 86.96 & 87.10 \\
\hline PB4 Ext & 83.85 & 81.91 & 88.27 & 89.11 & 85.32 & 82.28 & 86.89 & 86.98 \\
\hline PB5 & 86.45 & 83.57 & 90.64 & 92.79 & 86.53 & 83.30 & 84.98 & 89.07 \\
\hline PB6 & 86.16 & 82.87 & 88.46 & 90.86 & 85.83 & 82.50 & 85.53 & 87.42 \\
\hline PB7 & 86.83 & 82.96 & 90.60 & 92.79 & 85.82 & 82.50 & 84.72 & 88.70 \\
\hline PB8 & 85.78 & 82.50 & 88.71 & 90.98 & 86.37 & 83.33 & 85.26 & 86.84 \\
\hline
\end{tabular}

PB3 (C4 $\alpha-C 8$, catechin); PB4 (C4 $\alpha-C 8$, catechin-epicatechin); PB5 (C4 $\beta-C 6$, epicatechin); PB6 (C4 $\alpha-C 6$, catechin); PB7 (C4 $\beta-C 6$, epicatechin-catechin); PB8 (C4 $\alpha-\mathrm{C} 6$, catechin-epicatechin).

TABLE 4: Fukui indices of the procyanidin dimers calculated by the M05-2X/6-31G** DFT method using the Hirshfeld charge population analysis in an aqueous medium (SMD model).

\begin{tabular}{|c|c|c|c|c|}
\hline Procyanidin dimer & Atom/unit & Nucleophilic attack & Atom/unit & Electrophilic attack \\
\hline PB3 Com & $\mathrm{C}^{\prime} \mathrm{EU}$ & 0.095 & O5 TU & 0.085 \\
\hline PB3 Ext & $\mathrm{C} 4^{\prime} \mathrm{TU}$ & 0.097 & $\mathrm{O} 4^{\prime} \mathrm{EU}$ & 0.104 \\
\hline PB4 Com & $\mathrm{C} 4^{\prime} \mathrm{EU}$ & 0.095 & O5 TU & 0.088 \\
\hline PB4 Ext & $\mathrm{C}^{\prime}{ }^{\prime} \mathrm{TU}$ & 0.098 & O5 TU & 0.083 \\
\hline PB5 & $\mathrm{C}^{\prime} \mathrm{EU}$ & 0.105 & $\mathrm{O}^{\prime} \mathrm{EU}$ & 0.116 \\
\hline PB6 & $\mathrm{C} 4^{\prime} \mathrm{EU}$ & 0.096 & O7 TU & 0.056 \\
\hline PB7 & $\mathrm{C} 4^{\prime} \mathrm{EU}$ & 0.095 & O7 TU & 0.099 \\
\hline PB8 & $\mathrm{C} 4^{\prime} \mathrm{EU}$ & 0.097 & $\mathrm{O} 4^{\prime} \mathrm{EU}$ & 0.049 \\
\hline
\end{tabular}

PB3 (C4 $\alpha-C 8$, catechin); PB4 (C4 $\alpha-C 8$, catechin-epicatechin); PB5 (C4 $\beta-C 6$, epicatechin); PB6 (C4 $\alpha-C 6$, catechin); PB7 (C4 $\beta-C 6$, epicatechin-catechin); PB8 (C4 $\alpha-$ C6, catechin-epicatechin).

EU: extension unit; TU: terminal unit.

effective charges in the transition state, establishing sites susceptible to nucleophilic and electrophilic attack. According to the Fukui indices displayed in Table 4, the preferential site for nucleophilic attack in all PC dimers was the $\mathrm{C}^{\prime}$ atom (catechol group, ring B). For PB3 Com, PB4 Com, PB5, PB6, $\mathrm{PB} 7$, and $\mathrm{PB} 8$, this site was located in the extension unit (EU), while, for PB3 Ext and PB4 Ext, the site was located in the terminal unit (TU). These results are supported by the LUMO distribution (isovalue 0.02), which represents the molecular site for the positive charge density (Figure 4). The LUMO distribution also points out to other atoms that are potential sites for nucleophilic attack, but the charge density is mainly concentrated on ring $\mathrm{B}$, either of the extension unit or of the terminal unit, which is where the $\mathrm{C}^{\prime}$ atom is located.

The site for electrophilic attack was the most variable for the PC dimers. Table 4 shows that in PB3 Com, PB4 Com, PB4 Ext, PB6, and PB7 dimers, the electrophilic attack occurs preferentially either in the $\mathrm{O} 5$ or in $\mathrm{O} 7$, atoms that are part of the resorcinol moiety of ring A. The HOMO distribution in such molecules corroborates the electron density that is mainly concentered on ring $A$, especially in the terminal unit (Figure 5). In contrast, PB3 Ext, PB5, and PB8 showed as a preferential site for electrophilic attack, either $\mathrm{O}^{\prime}$ or $\mathrm{O} 4^{\prime}$, which are part of the catechol moiety of ring $\mathrm{B}$ in the extension unit.
The PB5 dimer proved to be the most susceptible for both nucleophilic and electrophilic attacks, since it presented the highest Fukui indices (0.105 and $0.116 \mathrm{eV}$, resp.). The PB3, PB4, PB6, PB7, and PB8 dimers showed susceptibilities for nucleophilic attack, similar to each other (Fukui indices 0.095-0.098 eV). However, the susceptibilities for electrophilic attack showed notable differences among these dimers (Fukui indices $0.049-0.116 \mathrm{eV}$ ), corresponding to the lowest value to the PB8 dimer.

\section{Conclusions}

The type of constituent units did not show a well-defined trend in relation to the effect that is exerted on the ability of PC dimers to transfer or capture electrons. Based on the analysis of the chemical potential and BDE, it is suggested that the factors with the most notable effects on the antioxidant properties of the PC dimers were the type of interflavan bond (C4-C8 or C4-C6) and the conformation (Com or Ext). Under this context, the antioxidant ability of the dimers PB3 and PB4 containing the interflavan bond $\mathrm{C} 4-\mathrm{C} 8$, in their conformation Com, was very similar to each other but greater than those of the dimers PB5, PB6, PB7, and PB8 containing the C4-C6 interflavan bond. It was also observed that PB8 showed the lowest antioxidant ability of all the analyzed PC 


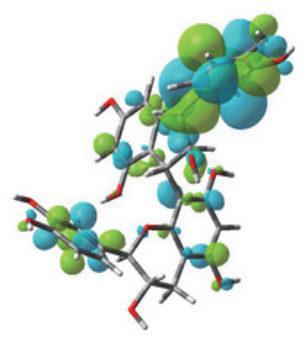

PB3 Com

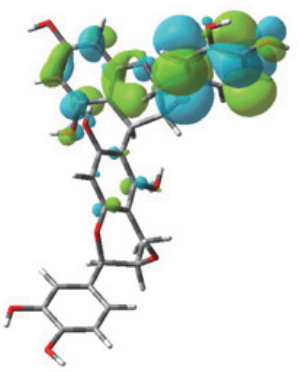

PB5

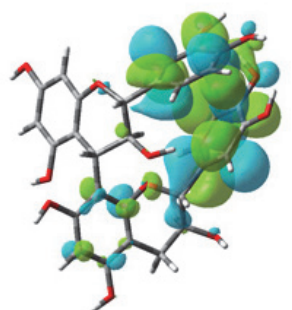

PB3 Ext

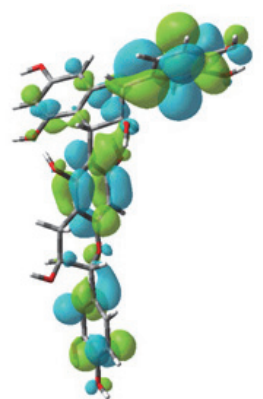

PB6

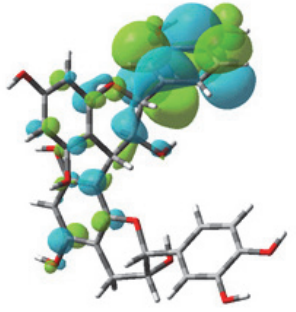

PB4 Com

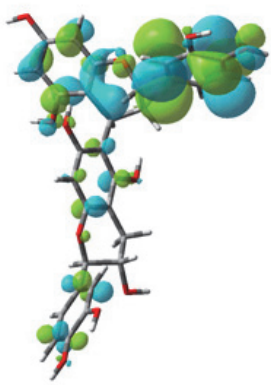

PB7

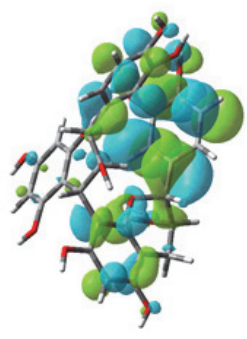

PB4 Ext

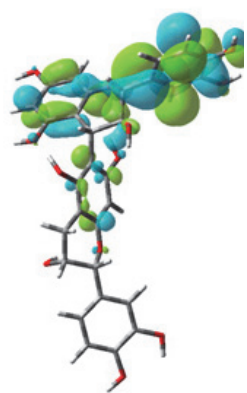

PB8

FIGURE 4: Charge distribution in the LUMO orbital of the procyanidin dimers optimized in aqueous medium (SMD model) using the M052X/6-31G ${ }^{* *}$ DFT method. Com: compact conformation; Ext: extended conformation.

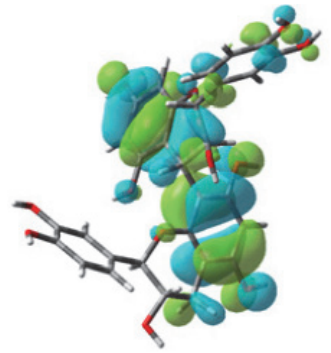

PB3 Com

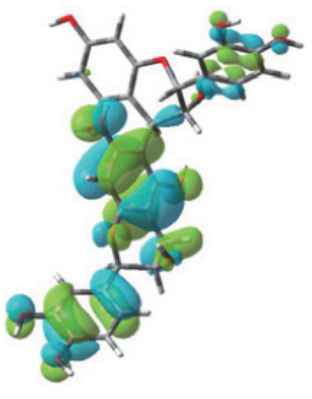

PB5

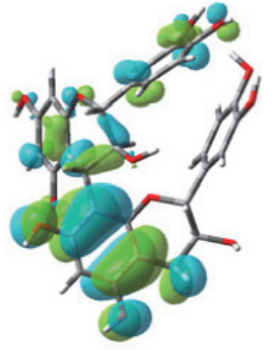

PB3 Ext

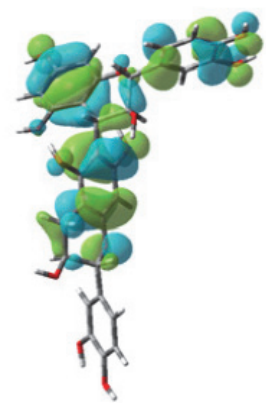

PB6

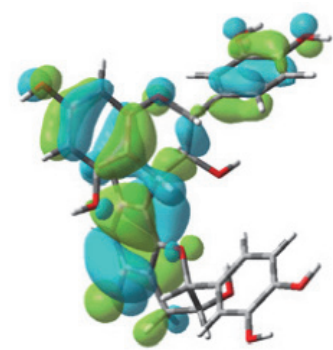

PB4 Com

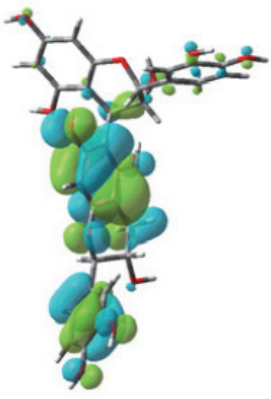

PB7

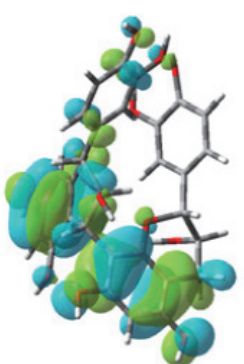

PB4 Ext

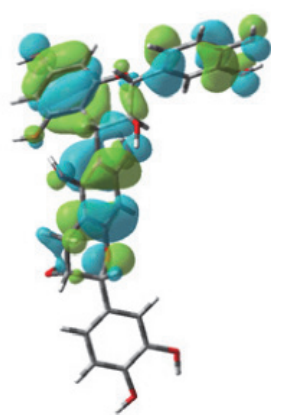

PB8

FIgURE 5: Charge distribution in the HOMO orbital of the procyanidin dimers optimized in aqueous medium (SMD model) using the M052X/6-31G** DFT method. Com: compact conformation; Ext: extended conformation.

dimers. Regarding the Fukui indices, the main differences between dimers were established on the base of their susceptibility to electrophilic attack, and the determinant factors were the conformation, together with the type and orientation of the interflavan bond. PB5, which contains the $\mathrm{C} 4 \beta-\mathrm{C} 6$ interflavan bond and acquires a conformation Com in its ground-state, showed greater susceptibility to electrophilic attack, while PB8, which contains the $\mathrm{C} 4 \alpha-\mathrm{C} 6$ interflavan bond and acquires a conformation Ext in its ground-state, showed the lowest susceptibility to electrophilic attack. 


\section{Competing Interests}

The authors of this paper declare that they have no competing interests.

\section{Acknowledgments}

The authors are very grateful to the Consejo Nacional de Ciencia y Tecnología (CONACYT), México, which financed the investigation under Grant no. CB2012-183739.

\section{References}

[1] O. Dangles, "Antioxidant activity of plant phenols: chemical mechanisms and biological significance," Current Organic Chemistry, vol. 16, no. 6, pp. 692-714, 2012.

[2] T. Enomoto, Y. Nagasako-Akazome, T. Kanda, M. Ikeda, and Y. Dake, "Clinical effects of apple polyphenols on persistent allergic rhinitis: a randomized double-blind placebo-controlled parallel arm study," Journal of Investigational Allergology and Clinical Immunology, vol. 16, no. 5, pp. 283-289, 2006.

[3] J. M. R. Da Silva, N. Darmon, Y. Fernandez, and S. Mitjavila, "Oxygen free radical scavenger capacity in aqueous models of different procyanidins from grape seeds," Journal of Agricultural and Food Chemistry ${ }^{\circledR}$, vol. 39, no. 9, pp. 1549-1552, 1991.

[4] I. Tarascou, M.-A. Ducasse, E. J. Dufourc et al., "Structural and conformational analysis of two native procyanidin trimers," Magnetic Resonance in Chemistry, vol. 45, no. 2, pp. 157-166, 2007.

[5] Y. Wang, S.-J. Chung, W. O. Song, and O. K. Chun, "Estimation of daily proanthocyanidin intake and major food sources in the U.S. diet," The Journal of Nutrition, vol. 141, no. 3, pp. 447-452, 2011.

[6] K. Ou and L. Gu, "Absorption and metabolism of proanthocyanidins," Journal of Functional Foods, vol. 7, no. 1, pp. 43-53, 2014.

[7] V. A. P. De Freitas, Y. Glories, and M. Laguerre, "Incidence of molecular structure in oxidation of grape seed procyanidins," Journal of Agricultural and Food Chemistry, vol. 46, no. 2, pp. 376-382, 1998.

[8] K. Klausen, A. G. Mortensen, B. Laursen, K. F. Haselmann, B. M. Jespersen, and I. S. Fomsgaard, "Phenolic compounds in different barley varieties: identification by tandem mass spectrometry (QStar) and NMR; quantification by liquid chromatography triple quadrupole-linear ion trap mass spectrometry (Q-Trap)," Natural Product Communications, vol. 5, no. 3, pp. 407-414, 2010.

[9] A. M. Mendoza-Wilson, S. I. Castro-Arredondo, A. EspinosaPlascencia, M. Del Refugio Robles-Burgueño, R. R. BalandránQuintana, and M. Del Carmen Bermúdez-Almada, "Chemical composition and antioxidant-prooxidant potential of a polyphenolic extract and a proanthocyanidin-rich fraction of apple skin," Heliyon, vol. 2, no. 2, Article ID e00073, 2016.

[10] P.-G. Pietta, "Flavonoids as antioxidants," Journal of Natural Products, vol. 63, no. 7, pp. 1035-1042, 2000.

[11] M. J. Frisch, G. W. Trucks, H. B. Schlegel et al., Gaussian 09, Revision A.1-SMP, Gaussian Inc, Wallingford, Conn, USA, 2009.

[12] Y. Zhao, N. E. Schultz, and D. G. Truhlar, "Design of density functionals by combining the method of constraint satisfaction with parametrization for thermochemistry, thermochemical kinetics, and noncovalent interactions," Journal of Chemical Theory and Computation, vol. 2, no. 2, pp. 364-382, 2006.

[13] L. A. Burns, Á. Vázquez-Mayagoitia, B. G. Sumpter, and C. D. Sherrill, "Density-functional approaches to noncovalent interactions: a comparison of dispersion corrections (DFT-D), exchange-hole dipole moment (XDM) theory, and specialized functionals," Journal of Chemical Physics, vol. 134, no. 8, Article ID 084107, 2011.

[14] A. V. Marenich, C. J. Cramer, and D. G. Truhlar, "Universal solvation model based on solute electron density and on a continuum model of the solvent defined by the bulk dielectric constant and atomic surface tensions," Journal of Physical Chemistry B, vol. 113, no. 18, pp. 6378-6396, 2009.

[15] E. F. Pettersen, T. D. Goddard, C. C. Huang et al., "UCSF Chimera-a visualization system for exploratory research and analysis," Journal of Computational Chemistry, vol. 25, no. 13, pp. 1605-1612, 2004.

[16] J. W. Ochterski, Thermochemistry in Gaussian, Gaussian, Inc., 2000.

[17] E. Lewars, Computacional Chemistry. Introduction to the Theory and Applications of Molecular and Quantum Mechanics, Kluwer Academic, Dordrecht, The Netherlands, 1st edition, 2003.

[18] P. Geerlings, F. De Proft, and W. Langenaeker, "Conceptual density functional theory," Chemical Reviews, vol. 103, no. 5, pp. 1793-1873, 2003.

[19] J. W. Ochterski, G. A. Petersson, and K. B. Wiberg, "A comparison of model chemistries," Journal of the American Chemical Society, vol. 117, no. 45, pp. 11299-11308, 1995.

[20] R. G. Parr and W. Yang, Density-Functional Theory of Atoms and Molecules, Oxford University Press, New York, NY, USA, 1989.

[21] W. Yang and W. J. Mortier, "The use of global and local molecular parameters for the analysis of the gas-phase basicity of amines," Journal of the American Chemical Society, vol. 108, no. 19 , pp. 5708-5711, 1986.

[22] M. L. Khan, E. Haslam, and M. P. Williamson, "Structure and conformation of the procyanidin B-2 dimer," Magnetic Resonance in Chemistry, vol. 35, no. 12, pp. 854-858, 1997.

[23] D. Lin-Vien, N. B. Colthup, W. G. Fateley, and J. G. Grasselli, "Alcohols and phenols," in The Handbook of Infrared and Raman Characteristic Frequencies of Organic Molecules, chapter 4, pp. 45-60, Academic Press, 1st edition, 1991.

[24] A. M. Mendoza-Wilson, S. I. Castro-Arredondo, and R. R. Balandrán-Quintana, "Computational study of the structurefree radical scavenging relationship of procyanidins," Food Chemistry, vol. 161, pp. 155-161, 2014.

[25] A. M. Mendoza-Wilson, F. J. Carmelo-Luna, H. AstiazaránGarcía, B. I. Pacheco-Moreno, I. Anduro-Corona, and M. L. Rascón-Durán, "DFT study of the physicochemical properties of A- and B-type procyanidin oligomers," Journal of Theoretical and Computational Chemistry, vol. 15, no. 8, Article ID 1650069, 18 pages, 2016.

[26] I. Tarascou, K. Barathieu, C. Simon et al., "A 3D structural and conformational study of procyanidin dimers in water and hydro-alcoholic media as viewed by NMR and molecular modeling," Magnetic Resonance in Chemistry, vol. 44, no. 9, pp. 868-880, 2006.

[27] H. M. Awad, M. G. Boersma, S. Boeren, P. J. Van Bladeren, J. Vervoort, and I. M. C. M. Rietjens, "The regioselectivity of glutathione adduct formation with flavonoid quinone/quinone methides is pH-dependent," Chemical Research in Toxicology, vol. 15, no. 3, pp. 343-351, 2002. 
[28] D. R. Roy, U. Sarkar, P. K. Chattaraj et al., "Analyzing toxicity through electrophilicity," Molecular Diversity, vol. 10, no. 2, pp. 119-131, 2006.

[29] A. M. Mendoza-Wilson and D. Glossman-Mitnik, "CHIH-DFT study of the electronic properties and chemical reactivity of quercetin," Journal of Molecular Structure: THEOCHEM, vol. 716, no. 1-3, pp. 67-72, 2005.

[30] A. M. Mendoza-Wilson, M. E. Armenta-Vázquez, S. I. CastroArredondo et al., "Potential of polyphenols from an aqueous extract of apple peel as inhibitors of free radicals: an experimental and computational study," Journal of Molecular Structure, vol. 1035, pp. 61-68, 2013.

[31] F. L. Hirshfeld, "Bonded-atom fragments for describing molecular charge densities," Theoretica Chimica Acta, vol. 44, no. 2, pp. 129-138, 1977. 

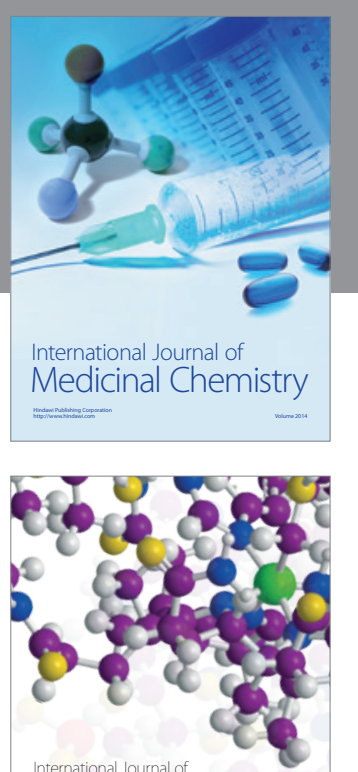

Carbohydrate Chemistry

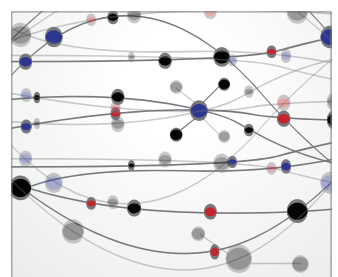

The Scientific World Journal
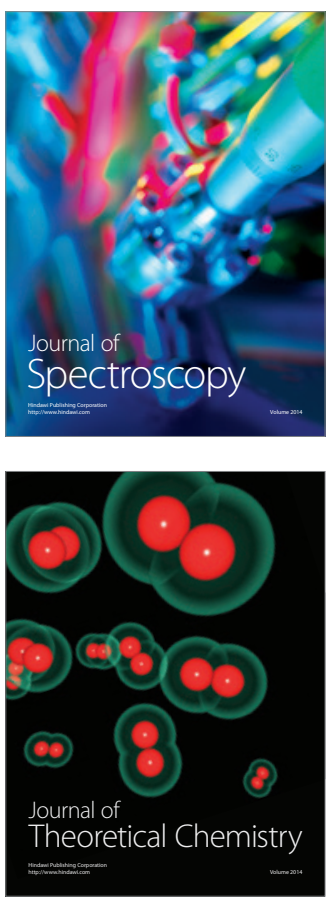
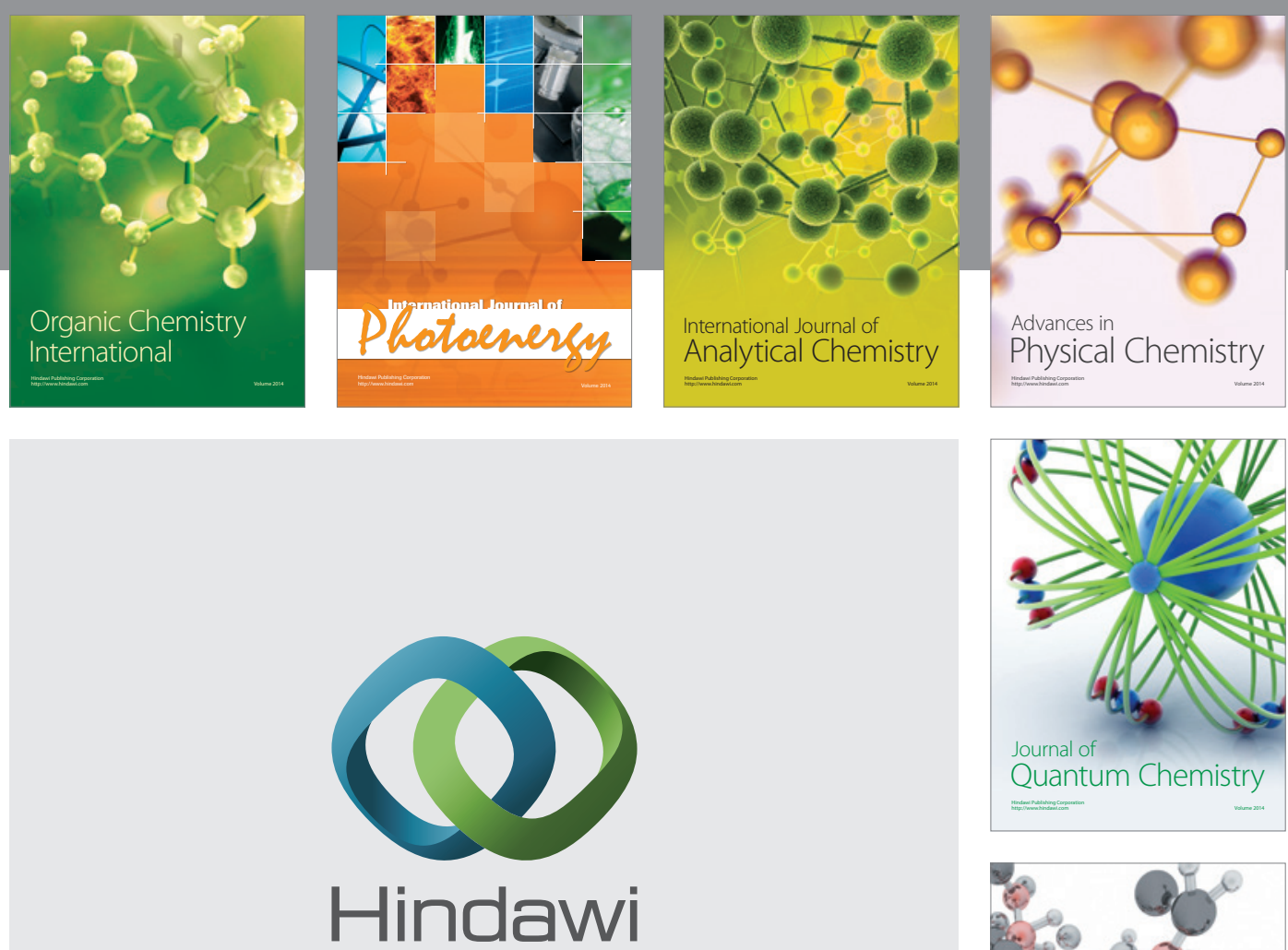

Submit your manuscripts at

https://www.hindawi.com

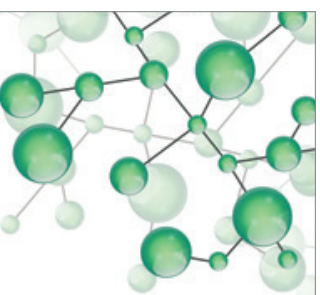

International Journal of

Inorganic Chemistry
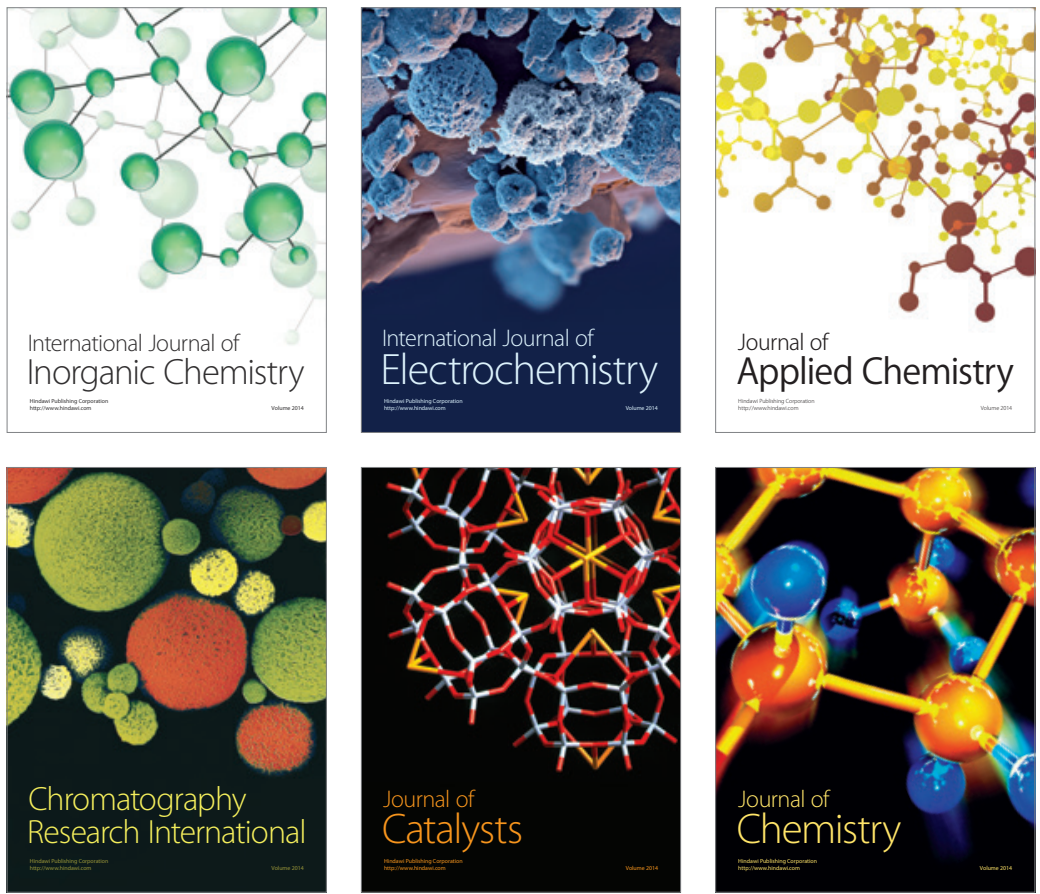

Journal of

Applied Chemistry
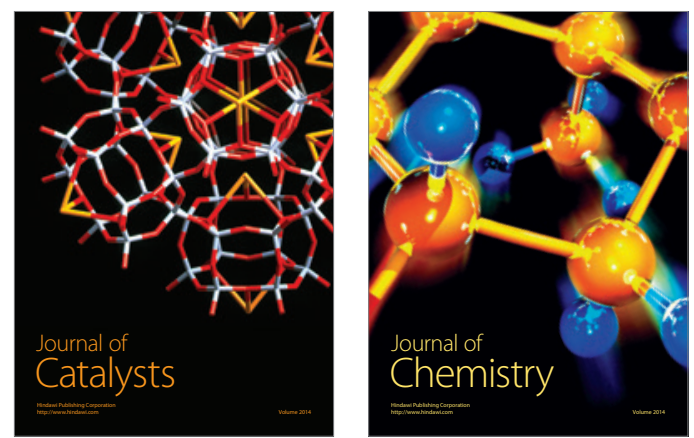
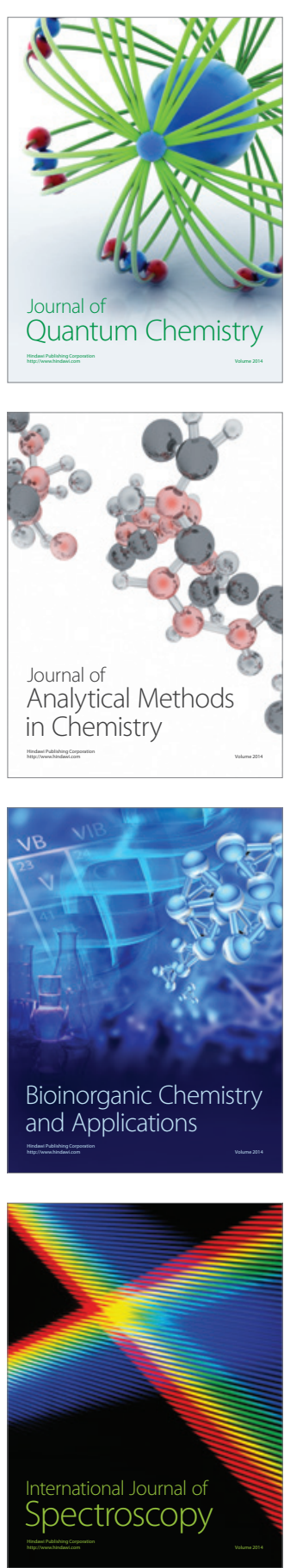